\title{
Bijections for inversion sequences, ascent sequences and 3-nonnesting set partitions
}

\author{
Sherry H. F. Yan \\ Department of Mathematics, Zhejiang Normal University, Jinhua 321004, P.R. China \\ huifangyan@hotmail.com
}

\begin{abstract}
Set partitions avoiding $k$-crossing and $k$-nesting have been extensively studied from the aspects of both combinatorics and mathematical biology. By using the generating tree technique, the obstinate kernel method and Zeilberger's algorithm, Lin confirmed a conjecture due independently to the author and MartinezSavage that asserts inversion sequences with no weakly decreasing subsequence of length 3 and enhanced 3-nonnesting partitions have the same cardinality. In this paper, we provide a bijective proof of this conjecture. Our bijection also enables us to provide a new bijective proof of a conjecture posed by Duncan and Steingrímsson, which was proved by the author via an intermediate structure of growth diagrams for 01-fillings of Ferrers shapes.
\end{abstract}

KEY WORDS: inversion sequence, ascent sequence, pattern avoiding, 3-nonnesting set partition.

AMS Mathematical Subject Classifications: 05A05, 05A19.

\section{Introduction}

Set partitions avoiding $k$-crossing and $k$-nesting have been extensively studied from the aspects of both combinatorics and mathematical biology; see [5, 6, 11] and the references therein. The objective of this paper is to provide a bijective proof of a conjecture due independently to the author [19] and Martinez-Savage [16], which was recently confirmed by Lin [13] using the generating tree technique, the obstinate kernel method [2] and Zeilberger's algorithm [17]. Our bijection also enables us to provide a new bijective proof of a conjecture posed by Duncan and Steingrímsson [8], which was proved by the author [19] via an intermediate structure of growth diagrams for 01-fillings of Ferrers shapes [11] and [12]. Let us first give an overview of the notation and terminology.

A sequence $x=x_{1} x_{2} \cdots x_{n}$ is said to be an inversion sequence of length $n$ if it satisfies $0 \leq x_{i}<i$ for all $1 \leq i \leq n$. Inversion sequences of length $n$ are in easy bijection with permutations of length $n$. An inversion sequence $x_{1} x_{2} \ldots x_{n}$ can be obtained from any permutation $\pi=\pi_{1} \pi_{2} \ldots \pi_{n}$ by setting $x_{i}=\left\{j \mid j<i\right.$ and $\pi_{j}>$ $\left.\pi_{i}\right\}$. 
Given a sequence of integers $x=x_{1} x_{2} \cdots x_{n}$, we say that the sequence $x$ has an ascent at position $i$ if $x_{i}<x_{i+1}$. The number of ascents of $x$ is denoted by $\operatorname{asc}(x)$. A sequence $x=x_{1} x_{2} \cdots x_{n}$ is said to be an ascent sequence of length $n$ if it satisfies $x_{1}=0$ and $0 \leq x_{i} \leq \operatorname{asc}\left(x_{1} x_{2} \cdots x_{i-1}\right)+1$ for all $2 \leq i \leq n$. Ascent sequences were introduced by Bousquet-Mélou et al. [3] in their study of $(2+2)$-free posets, which are closely connected to unlabeled $(2+2)$-free posets, permutations avoiding a certain pattern, and a class of involutions introduced by Stoimenow [18]. We call an ascent sequence with no two consecutive equal entries a primitive ascent sequence.

Pattern avoiding permutations have been extensively studied over last decade. For a thorough summary of the current status of research, see Bóna's book [1] and Kitaev's book [10. Analogous to pattern avoidance on permutations, CorteelMartinez-Savage-Weselcouch [7] and Mansour-Shattuck [15] initiated the study of inversion sequences avoiding certain pattern. Pattern avoiding inversion sequences are closely related to Catalan numbers, large Schröder numbers, Euler numbers and Baxter numbers (see [7], [9], [13] and [15]). In their paper [8], Duncan and Steingrímsson studied ascent sequences avoiding certain patterns. Further results on the enumeration of pattern-avoiding ascent sequences could be found in [4, 14, 19]. By using the generating tree technique, the obstinate kernel method and Zeilberger's algorithm, Lin [13] confirmed the following conjecture proposed by Martinez-Savage [16].

Conjecture 1.1 ( Martinez-Savage [16] ) Inversion sequences of length $n$ and with no weakly decreasing subsequence of length 3 are equinumerous with enhanced 3nonnesting (3-noncrossing) set partitions of $[n]$.

As remarked by Lin [13], this conjecture has already been proposed by Yan [19] in the the course of confirming the following conjecture posed by Duncan and Steingrímsson [8].

Conjecture 1.2 ( See [8], Conjecture 3.3) Ascent sequences of length $n$ and with no decreasing subsequence of length 3 are equinumerous with 3-nonnesting (3-noncrossing) set partitions of $[n]$.

Recall that a subsequence $x_{i_{1}} x_{i_{2}} \ldots x_{i_{k}}$ of a sequence $x=x_{1} x_{2} \ldots x_{n}$ is said to be decreasing if $i_{1}<i_{2}<\ldots<i_{k}$ and $x_{i_{1}}>x_{i_{2}}>\ldots>x_{i_{k}}$ and to be weakly decreasing if $i_{1}<i_{2}<\ldots<i_{k}$ and $x_{i_{1}} \geq x_{i_{2}} \geq \ldots \geq x_{i_{k}}$. Denote by $\mathcal{A}_{k}(n)$ and $\mathcal{P} \mathcal{A}_{k}(n)$ the set of ordinary and primitive ascent sequences of length $n$ and with no decreasing subsequence of length $k$, respectively. Let $\mathcal{I}_{k}(n)$ denote the set of inversion sequences of length $n$ and with no weakly decreasing sequences of length $k$. 
A set partition $P$ of $[n]=\{1,2, \cdots, n\}$ can be represented by a diagram with vertices drawn on a horizontal line in increasing order. For a block $B$ of $P$, we write the elements of $B$ in increasing order. Suppose that $B=\left\{i_{1}, i_{2}, \cdots, i_{k}\right\}$. Then we draw an arc from $i_{1}$ to $i_{2}$, an arc from $i_{2}$ to $i_{3}$, and so on. Such a diagram is called the linear representation of $P$, see Figure 1 for example. The enhanced representation of $P$ is defined to the union of the standard representation of $P$ and the set of loops $(i, i)$, where $i$ ranges over all the singleton blocks $\{i\}$ of $P$. Then one defines a $k$-crossing of a set partition to be a subset $\left\{\left(i_{1}, j_{1}\right),\left(i_{2}, j_{2}\right), \cdots,\left(i_{k}, j_{k}\right)\right\}$ of its linear representation where $i_{1}<i_{2}<\cdots<i_{k}<j_{1}<j_{2}<\cdots<j_{k}$, and an enhanced $k$-crossing of a set partition to be a subset $\left\{\left(i_{1}, j_{1}\right),\left(i_{2}, j_{2}\right), \cdots,\left(i_{k}, j_{k}\right)\right\}$ of its enhanced representation where $i_{1}<i_{2}<\cdots<i_{k} \leq j_{1}<j_{2}<\cdots<j_{k}$. A partition without any (enhanced) $k$-crossings is said to be (enhanced ) $k$-noncrossing. Similarily, a $k$-crossing is defined to be a subset $\left\{\left(i_{1}, j_{1}\right),\left(i_{2}, j_{2}\right), \cdots,\left(i_{k}, j_{k}\right)\right\}$ of its enhanced representation where $i_{1}<i_{2}<\cdots<i_{k}<j_{k}<j_{k-1}<\cdots<j_{1}$, and an enhanced $k$-nesting is defined to be a subset $\left\{\left(i_{1}, j_{1}\right),\left(i_{2}, j_{2}\right), \cdots,\left(i_{k}, j_{k}\right)\right\}$ of its enhanced representation where $i_{1}<i_{2}<\cdots<i_{k} \leq j_{k}<j_{k-1}<\cdots<j_{1}$. A set partition without any (enhanced ) $k$-nestings is said to be (enhanced) $k$ nonnesting. Chen et al. [5] proved that (enhanced) $k$-nonnesting set partitions of $[n]$ are equinumerous with (enhanced) $k$-noncrossing set partitions of $[n]$ bijectively using hesitating tableaux as an intermediate object. Denote by $\mathcal{C}_{k}(n)$ and $\mathcal{E}_{k}(n)$ the set of ordinary and enhanced $k$-nonnesting set partitions of $[n]$, respectively.

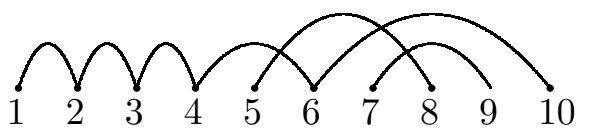

Figure 1: The linear representation of a set partition $\pi=$ $\{\{1,2,3,4,6,10\},\{5,8\},\{7,9\}\}$.

\section{$2 \quad$ Bijective proof of Conjecture 1.1}

In this section, we shall provide a bijective proof of Conjecture 1.1 by showing that inversion sequences of length $n$ and with no weakly decreasing subsequence of length 3 are in bijection with enhanced 3-nonnesting partitions of $[n]$. To this end, we recall some necessary notation and terminology.

A triangular shape of order $n$ is the left-justified array of $\left(\begin{array}{c}n+1 \\ 2\end{array}\right)$ squares in which the $i$ th row contains exactly $i$ squares. Let $\Delta_{n}$ be the triangular shape of order $n$. In a triangular shape, we number rows from top to bottom and columns from left to right and identify squares using matrix coordinate. The $i$ th row (column) is 
called row (column) $i$. For example, the square in the first row and second column is numbered $(1,2)$.

A 01-filling of a triangular shape $\Delta_{n}$ is obtained by filling the squares of $\Delta_{n}$ with $1^{\prime} s$ and $0^{\prime} s$, see Figure 2 for example, where we represent a 1 by a $\bullet$ and suppress the 0's. A 01-filling of a triangular shape is said to be valid if every row contains at most one 1. A row (column) of a 01-filling is said to a zero if all the squares at this row ( column) are filled with $0^{\prime} s$. A $N E$-chain of a 01-filling is a sequence of $1^{\prime} s$ such that any 1 is strictly above and weakly to the right of the preceding 1 in the sequence. For example, in Figure 2, the sequence of $1^{\prime} s$ lying in the squares $(6,3)$, $(5,4$,$) and (4,4)$ form a NE-chain of length 3.

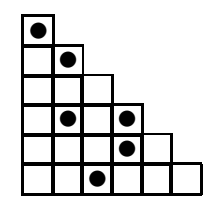

Figure 2: An example of a 01-filling of a triangular shape of order 6 .

An inversion sequence $x_{1} x_{2} \ldots x_{n}$ can be encoded by a 01-filling of $\Delta_{n}$ in which the square $\left(i, x_{i}+1\right)$ is filled with a 1 for all $1 \leq i \leq n$ and all the other squares are filled with $0^{\prime} s$. It is easily seen that a weakly decreasing sequence of length $k$ corresponds to a NE-chain of length $k$. Denote by $\mathcal{M}_{k}(n)$ the set of 01-fillings of $\Delta_{n}$ with the property that every row contains exactly one 1 and there is no NE-chain of length $k$.

Theorem 2.1 There is a one-to-one correspondence between the set $\mathcal{I}_{k}(n)$ and the set $\mathcal{M}_{k}(n)$.

In his paper [11, Krattenthaler established a bijection between set partitions of $[n]$ and 01-fillings of $\Delta_{n}$ in which every row and every column contain at most one 1 , and either column $i$ or row $i$ contains at least one 1 for all $1 \leq i \leq n$. For the sake of completeness, we give a brief description of this bijection. Given a set partition $\pi$ of $[n]$, we can get a 01 -filling of $\Delta_{n}$ by putting a 1 in the square $(j, i)$ if $(i, j)$ is an arc in its enhanced representation, and, in addition, by putting a 1 in the the square $(i, i)$ if $(i, i)$ is a loop in its enhanced representation. The 01-filling corresponding to the set partition $\pi=\{\{1,3,6\},\{2,8\},\{4\},\{5,7,9\}\}$ is indicated in Figure 3 . From the construction of Krattenthaler's bijection, an enhanced $k$-nesting of a set partition corresponds to a NE-chain of length $k$ in its corresponding 01-filling.

Denote by $\mathcal{N}_{k}(n)$ the set of 01-fillings of $\Delta_{n}$ satisfying the following properties: 
(a1) every row and every column contain at most one 1;

(b1) either column $i$ or row $i$ contains at least one 1 for all $1 \leq i \leq n$;

(c1) there is no NE-chain of length $k$.

From Krattenthaler's bijection, we immediately get the following result.

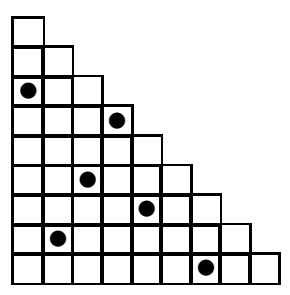

Figure 3: A set partition $\pi=\{\{1,3,6\},\{2,8\},\{4\},\{5,7,9\}\}$ and its corresponding 01-filling.

Theorem 2.2 The 01-fillings of the set $\mathcal{E}_{k}(n)$ are in bijection with the 01-fillings of the set $\mathcal{N}_{k}(n)$.

In view of Theorems 2.1 and 2.2, in order to prove Conjecture 1.1, it suffices to establish a bijection between the set $\mathcal{M}_{k}(n)$ and the set $\mathcal{N}_{k}(n)$. To this end, we define two transformations, which will play an essential role in the construction of the bijection.

The transformation $\alpha$ Let $F$ be a valid 01-filling of $\Delta_{n}$ without any NE-chain of length 3. If every column of $F$ contains at most one 1, we simply define $\alpha(F)=F$. Otherwise, find the leftmost column $i$ which contains at least two $1^{\prime} s$. Suppose the square $(i, j)$ is filled with a 1 for some $1 \leq j \leq i$. Assume that the $1^{\prime} s$ below and weakly to the left of the square $(i, i)$ are positioned at the squares $\left(r_{1}, c_{1}\right)$, $\left(r_{2}, c_{2}\right), \ldots,\left(r_{m}, c_{m}\right)$ with $r_{1}<r_{2}<\ldots<r_{m}$. Let $r_{0}=i, c_{0}=j$. Suppose that the topmost 1 in column $i$ is at row $r_{s}$.

If row $r_{s}+1$ contains a 1 which is to the right of the square $\left(r_{s}, c_{s}\right)$, then define $\alpha(F)$ to be the 01-filling of $\Delta_{n}$ obtained from $F$ by the following procedure:

- For all $0 \leq \ell \leq s$, replace the 1 at the square $\left(r_{\ell}, c_{\ell}\right)$ with a 0 ;

- For all $0 \leq \ell<s$, fill the square $\left(r_{\ell+1}, c_{\ell}\right)$ with a 1 ; 
- Leave all the other squares fixed.

Otherwise, define $\alpha(F)$ to be the 01-filling of $\Delta_{n}$ obtained from $F$ by the following procedure:

- For all $0 \leq \ell \leq m$, replace the 1 at the square $\left(r_{\ell}, c_{\ell}\right)$ with a 0 ;

- For all $0 \leq \ell<m$, fill the square $\left(r_{\ell+1}, c_{\ell}\right)$ with a 1 ;

- Leave all the other squares fixed.

Now we proceed to show that the transformation $\alpha$ has the following desired properties.

Lemma 2.3 In $\alpha(F)$, each column to the left of column $i$ contains at most one 1 and column $i$ contains exactly one 1 .

Proof. It is obvious from the selection of column $i$ and the construction of the transformation $\alpha$.

Lemma 2.4 The filling $\alpha(F)$ is a valid 01-filling of $\Delta_{n}$ containing no NE-chain of length 3 .

Proof. According to the construction of the transformation $\alpha$, it is easily seen that $\alpha(F)$ is a valid 01-filling of $\Delta_{n}$. Now we proceed to show that $\alpha(F)$ contains no NE-chain of length 3 . If not, suppose that the $1^{\prime} s$ positioned at the squares $\left(a_{1}, b_{1}\right)$, $\left(a_{2}, b_{2}\right)$ and $\left(a_{3}, b_{3}\right)$ form a NE-chain of length 3 , where $a_{1}<a_{2}<a_{3}$. Since $F$ has no NE-chain of length 3 , the square $\left(a_{1}, b_{1}\right)$ must be positioned below and to the right of the square $\left(r_{s}, c_{s}\right)$. Suppose that there exists a 1 at row $r_{s}+1$ which is to the right of the square $\left(r_{s}, c_{s}\right)$. From the construction of $\alpha(F)$, it is easy to check that all the squares below row $r_{s}$ remain the same as those of $F$. This implies that there is no NE-chain of length 3 below row $r_{s}$ in $\alpha(F)$. This contradicts the fact that $\left(a_{1}, b_{1}\right)$ is below row $r_{s}$. Hence, row $r_{s}+1$ does not contain a 1 which is to the right of the square $\left(r_{s}, c_{s}\right)$. This implies that the square $\left(a_{1}, b_{1}\right)$ is below and to the right of the square $\left(r_{s+1}, c_{s}\right)$. Since $F$ has no NE-chain of length 3 , there is no NE-chain of length 2 below and to the left of the square $\left(r_{s+1}, c_{s}\right)$ in $\alpha(F)$. This yields that both the square $\left(a_{1}, b_{1}\right)$ and the square $\left(a_{2}, b_{2}\right)$ are positioned to the right of the square $\left(r_{s}, c_{s}\right)$. From the fact that $F$ contains no NE-chain of length 3 , we have $c_{s}=c_{m}=i$. Then the $1^{\prime} s$ positioned at the squares $\left(a_{1}, b_{1}\right),\left(a_{2}, b_{2}\right)$ and $\left(r_{m}, c_{m}\right)$ would form a NE-chain of length 3 in $F$, which contradicts the hypothesis. This completes the proof. 
Lemma 2.3 states that the column $i$ that we find in the transformation $\alpha$ can only go right. Hence, there will be no column containing at least two $1^{\prime} s$ in the resulting filling after finitely many iterations of $\alpha$. Lemma 2.4 tells us that the resulting filling is a valid 01-filling of $\Delta_{n}$ containing no NE-chain of length 3 . Therefore, we will get a 01-filling in $N_{3}(n)$ after finitely applying many iterations of $\alpha$ to a 01-filling $F$ in $\mathcal{M}_{3}(n)$. Define $\phi(F)$ to be the resulting filling. Figure 4 illustrates an example of two iterations of $\alpha$ to a 01-filling in $\mathcal{M}_{3}(9)$.

The transformation $\beta$ Let $F$ be a valid filling of $\Delta_{n}$ which verifies property (b1) and contains no NE-chain of length 3 . If every row contains a 1 in $F$, then we simply define $\beta(F)=F$. Otherwise, find the lowest zero row $i$. Suppose that the $1^{\prime} s$ below and weakly to the left of the square $(i, i)$ are positioned at the squares $\left(r_{1}, c_{1}\right),\left(r_{2}, c_{2}\right), \ldots,\left(r_{m}, c_{m}\right)$ with $r_{1}<r_{2}<\ldots<r_{m}$. Assume that $r_{0}=i$. Suppose that the topmost 1 at column $i$ is positioned at the square $\left(r_{s}, c_{s}\right)$.

If there is at least one 1 which is above and to the right of the square $\left(r_{s}, c_{s}\right)$, then find the topmost square, say $(p, q)$, containing such a 1 . Then we have $p=r_{t}+1$ for some $0 \leq t \leq s-1$. Define $\beta(F)$ to be the 01-filling of $\Delta_{n}$ obtained from $F$ by the following procedure:

- For all $1 \leq \ell \leq t$, replace the 1 at the square $\left(r_{\ell}, c_{\ell}\right)$ with a 0 ;

- For all $0 \leq \ell \leq t$, fill the square $\left(r_{\ell}, c_{\ell+1}\right)$ with a 1 with the assumption $c_{t+1}=i$;

- Leave all the other squares fixed.

Otherwise, define $\beta(F)$ to be the 01-filling of $\Delta_{n}$ obtained from $F$ by the following procedure:

- For all $1 \leq \ell \leq m$, replace the 1 at the square $\left(r_{\ell}, c_{\ell}\right)$ with a 0 ;

- For all $0 \leq \ell \leq m$, fill the square $\left(r_{\ell}, c_{\ell+1}\right)$ with a 1 with the assumption $c_{m+1}=i$

- Leave all the other squares fixed.

Now we proceed to show that the transformation $\beta$ has the following analogous properties of $\alpha$.

Lemma 2.5 In $\beta(F)$, every row below row $i$ (including row $i$ ) contains exactly one 1.

Proof. It is obvious from the selection of row $i$ and the construction of the transformation $\beta$. 
Lemma 2.6 The filling $\beta(F)$ is a valid 01-filling of $\Delta_{n}$ which verifies property (b1) and contains no NE-chain of length 3.

Proof. It is obvious that $\beta(F)$ is a valid 01-filling of $\Delta_{n}$ which verifies property (b1). Now we proceed to show that $\beta(F)$ contains no NE-chain of length 3. If not, suppose that the $1^{\prime} s$ positioned at the squares $\left(a_{1}, b_{1}\right),\left(a_{2}, b_{2}\right)$ and $\left(a_{3}, b_{3}\right)$ form a NE-chain of length 3 , where $a_{1}<a_{2}<a_{3}$.

Suppose that there is at least one 1 which is above and to the right of the square $\left(r_{s}, c_{s}\right)$ in $F$. In this case, all the squares below row $r_{t}$ in $\beta(F)$ remain the same as those of $F$. Since $F$ contains no NE-chain of length 3 , we must have $\left(a_{1}, b_{1}\right)=\left(r_{t}, i\right)$. Hence, the $1^{\prime} s$ positioned at the squares $(p, q),\left(a_{2}, b_{2}\right)$ and $\left(a_{3}, b_{3}\right)$ form a NE-chain of length 3 , which contradicts the hypothesis. Thus, $F$ does not contain a 1 which is above and to the right of the square $\left(r_{s}, c_{s}\right)$. According to the construction of $\beta(F)$, one of $\left(a_{1}, b_{1}\right),\left(a_{2}, b_{2}\right)$ and $\left(a_{3}, b_{3}\right)$ must fall in $\left(r_{m}, i\right)$. Since there is no 1 which is below row $r_{m}$ and to the left of column $i$ in $\beta(F)$, we have $\left(a_{3}, b_{3}\right)=\left(r_{m}, i\right)$. Then the $1^{\prime} s$ positioned at the squares $\left(a_{1}, b_{1}\right),\left(a_{2}, b_{2}\right)$ and $\left(r_{m}, i\right)$ would form a NE-chain of length 3 in $F$, which contradicts the hypothesis. This completes the proof.

Lemma 2.5 states that the row $i$ that we find in the transformation $\beta$ can only go upside. Hence, there will be no zero row after finitely many iterations of $\beta$. Lemma 2.6 tells us that the resulting filling is a valid 01-filling of $\Delta_{n}$ containing no NE-chain of length 3 . Hence, we will get a 01-filling in $\mathcal{M}_{3}(n)$ after finitely applying many iterations of $\beta$ to a 01-filling $F$ in $\mathcal{N}_{3}(n)$. Define $\psi(F)$ to be the resulting filling.

Theorem 2.7 The maps $\phi$ and $\psi$ induce a bijection between the set $\mathcal{M}_{3}(n)$ and the set $\mathcal{N}_{3}(n)$.

Proof. It suffices to show that the maps $\phi$ and $\psi$ are inverses of each other. First, we proceed to show that $\phi$ is the inverse of the map $\psi$, that is, $\phi(\psi(F))=F$ for any 01-filling $F \in \mathcal{N}_{3}(n)$. To this end, it suffices to show that $\alpha\left(\beta^{k}(F)\right)=\beta^{k-1}(F)$. Suppose that at the $k$ th application of $\beta$ to $\beta^{k-1}(F)$, the selected row is row $i$. Suppose that the $1^{\prime} s$ below and weakly to the left of the square $(i, i)$ are positioned at the squares $\left(r_{1}, c_{1}\right),\left(r_{2}, c_{2}\right), \ldots,\left(r_{m}, c_{m}\right)$ with $r_{1}<r_{2}<\ldots<r_{m}$. Assume that $r_{0}=i$. Suppose that the topmost 1 at column $i$ is positioned at the square $\left(r_{s}, c_{s}\right)$. We have two cases.

If there is at least one 1 which is above and to the right of the square $\left(r_{s}, c_{s}\right)$ in $\beta^{k-1}(F)$, then find the topmost square $(p, q)$ containing such a 1 . Assume that $p=r_{t}+1$ for some $0 \leq t<s$. From the construction of the transformation $\beta$, the square $\left(r_{\ell}, c_{\ell+1}\right)$ of $\beta^{k}(F)$ is filled with a 1 for all $1 \leq \ell \leq t$ with the assumption $c_{t+1}=i$ and all the other squares remain the same as those of $\beta^{k-1}(F)$. Clearly, in 
$\beta^{k}(F)$, all the columns to the left of column $i$ contains at most one 1 , and column $i$ contains exactly two $1^{\prime} s$. Hence, when we apply the transformation $\alpha$ to $\beta^{k}(F)$, the column that we select is just column $i$ and the topmost 1 at column $i$ is positioned at the square $\left(r_{t}, i\right)$. Moreover, the 1 positioned at the square $(p, q)$ is to the right of square $\left(r_{t}, i\right)$ and $p=r_{t}+1$. From the construction of $\alpha$, it is not dificult to check that $\alpha\left(\beta^{k}(F)\right)=\beta^{k-1}(F)$.

If there does not exists any 1 which is above and to the right of the square $\left(r_{s}, c_{s}\right)$ in $\beta^{k-1}(F)$, From the construction of the transformation $\beta$, the square $\left(r_{\ell}, c_{\ell+1}\right)$ of $\beta^{k}(F)$ is filled with a 1 for all $1 \leq \ell \leq m$ with the assumption $r_{m+1}=i$ and all the other squares remain the same as those of $\beta^{k-1}(F)$. Clearly, in $\beta^{k}(F)$, all the columns to the left of column $i$ contains at most one 1 , in which column $i$ contains exactly two $1^{\prime} s$. Hence, when we apply the transformation $\alpha$ to $\beta^{k}(F)$, the column that we select is just column $i$ and the topmost 1 at column $i$ is positioned at the square $\left(r_{s-1}, c_{s}\right)$. Notice that there does not exist any 1 which is above and to the right of square $\left(r_{s}, c_{s}\right)$ in $\beta^{k-1}(F)$. Hence, there is no 1 at row $r_{s-1}+1$ which is to the right of the square $\left(r_{s-1}, c_{s}\right)$. From the construction of $\alpha$, it is easily seen that $\alpha\left(\beta^{k}(F)\right)=\beta^{k-1}(F)$.

Combining the two above cases, we have deduced that $\alpha\left(\beta^{k}(F)\right)=\beta^{k-1}(F)$. By similar arguments, one can verify that $\beta\left(\alpha^{k}(F)\right)=\alpha^{k-1}(F)$ for any 01-filling $F$ of $\mathcal{M}_{3}(n)$. The details are omitted here. Hence, the maps $\phi$ and $\psi$ are inverses of each other. Thus, the maps $\phi$ and $\psi$ induce a bijection between the set $\mathcal{M}_{3}(n)$ and the set $\mathcal{N}_{3}(n)$ as claimed.

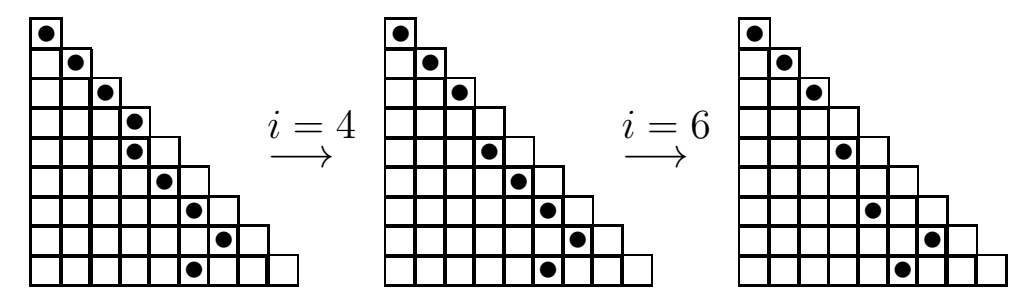

Figure 4: An example of two iterations of $\alpha$ to a 01-filling in $\mathcal{M}_{3}(9)$.

Combining Theorems 2.1, 2.2 and 2.7, we are led to a bijective proof of Conjecture 1.1 . 


\section{$3 \quad$ Bijective proof of Conjecture 1.2}

In this section, we shall give a new bijective proof of Conjecture 1.2 relying on the bijection $\phi$.

In the following, a 01-filling in $\mathcal{M}_{3}(n)$ will be identified with a sequence $\left\{\left(1, a_{1}\right)\right.$, $\left.\left(2, a_{2}\right), \cdots,\left(n, a_{n}\right)\right\}$, where $1 \leq a_{i} \leq i$ and $a_{i}=k$ if and only if there is a 1 in the $i$ th row and $k$ th column. In the course of proving Conjecture [1.2, Yan [19] provided a bijection $\gamma$ between the set $\mathcal{P} \mathcal{A}_{3}(n+1)$ and the set $\mathcal{M}_{3}(n)$. Let $x=$ $x_{1} x_{2} \cdots x_{n+1} \in \mathcal{P} \mathcal{A}_{3}(n+1)$. Define $\gamma(x)=\left\{\left(1, a_{1}\right),\left(2, a_{2}\right), \cdots,\left(n, a_{n}\right)\right\}$ where $a_{i}=i+x_{i+1}-\operatorname{asc}\left(x_{1} x_{2} \cdots x_{i+1}\right)$ for all $i=1,2, \cdots, n$. For example, let $x=$ $012340415 \in \mathcal{P} \mathcal{A}_{3}(9)$. Then we have

$$
\gamma(x)=\{(1,1),(2,2),(3,3),(4,4),(5,1),(6,5),(7,3),(8,7)\} .
$$

The inverse of the map $\gamma$ is defined as follows. Let $F=\left\{\left(1, a_{1}\right),\left(2, a_{2}\right), \cdots,\left(n, a_{n}\right)\right\}$. Define $\gamma^{-1}(F)=\left(x_{1}, x_{2}, \cdots, x_{n+1}\right)$ inductively as follows:

- $x_{1}=0$ and $x_{2}=1$;

- if $a_{i-1}<a_{i}$, then $x_{i+1}=\operatorname{asc}\left(x_{1} x_{2} \cdots x_{i}\right)+1+a_{i}-i$ for all $2 \leq i \leq n$;

- if $a_{i-1} \geq a_{i}$, then $x_{i+1}=\operatorname{asc}\left(x_{1} x_{2} \cdots x_{i}\right)+a_{i}-i$ for all $2 \leq i \leq n$.

Recall that Krattenthaler [11] also established a bijection between set partitions of $[n+1]$ and 01-fillings of $\Delta_{n}$ in which every row and every column contain at most one 1 . Given a set partition $\pi$ of $[n]$, we can get a 01 -filling of $\Delta_{n}$ by putting a 1 in the square $(j-1, i)$ if $(i, j)$ is an arc in its linear representation. From the construction of Krattenthaler's bijection, a $k$-nesting of a set partition corresponds to a NE-chain of length $k$ in its corresponding 01-filling.

Denote by $\mathcal{P}_{k}(n)$ the set of 01-fillings of $\Delta_{n}$ in which every row and every column contain at most one 1 , and there is no NE-chain of length $k$.

The following result follows immediately from Krattenthaler's bijection [1].

Theorem 3.1 There is a one-to-one correspondence between the set $\mathcal{C}_{k}(n+1)$ and the set $\mathcal{P}_{k}(n)$.

By Theorem 3.1, in order to provide a bijection between $\mathcal{A}_{3}(n)$ and $C_{3}(n)$, it suffices to establish a bijection between the set $\mathcal{A}_{3}(n+1)$ and the set $\mathcal{P}_{3}(n)$.

In a 01-filling, if both row $i$ and column $i$ are zero, then row (column) $i$ is said to be critical. 
Theorem 3.2 There is a bijection between the set $\mathcal{A}_{3}(n+1)$ and the set $\mathcal{P}_{3}(n)$.

Proof. First we shall describe a map $\delta$ from the set $\mathcal{A}_{3}(n+1)$ to the set $\mathcal{P}_{3}(n)$. Let $x \in \mathcal{A}_{3}(n+1)$. It is apparent that the ascent sequence $x$ can be written as $x_{1}^{c_{1}} x_{2}^{c_{2}} \cdots x_{k+1}^{c_{k+1}}$, where $x_{i} \neq x_{i+1}$ and $c_{i} \geq 1$ for all $i \geq 1$. Let $x^{\prime}=x_{1} x_{2} \cdots x_{k+1}$. Obviously, $x^{\prime}$ is a primitive ascent sequence in $\mathcal{P A}_{3}(k+1)$. Let $F=\gamma\left(x^{\prime}\right)$ and $F^{\prime}=\phi(F)$. Clearly, we have $F \in \mathcal{M}_{3}(k)$ and $F^{\prime} \in \mathcal{N}_{3}(k)$. Now we can generate a 01-filling $F^{\prime \prime}$ of $\Delta_{n}$ from $F^{\prime}$ by inserting $c_{1}-1$ consecutive zero rows immediately above row 1 and $c_{1}-1$ consecutive zero columns immediately to the left of column 1 , and inserting $c_{i}$ consecutive zero rows immediately below row $i$ and $c_{i}$ consecutive zero columns immediately to the right of column $i$ for all $1 \leq i \leq k$. Define $\delta(x)=F^{\prime \prime}$. It is not difficult to see that the resulting filling $F^{\prime \prime}$ is an element of $\mathcal{P}_{3}(n)$. This implies that the map $\delta$ is well defined.

In order to prove that $\delta$ is a bijection, we construct a map $\delta^{\prime}$ from the set $\mathcal{P}_{3}(n)$ to the set $\mathcal{A}_{3}(n+1)$. Given a 01 -filling $F \in \mathcal{P}_{3}(n)$, we can recover an ascent sequence $\delta^{\prime}(F)$ as follows. Suppose that there are $k$ non-critical rows in $F$. Let rows $i_{1}$, $i_{2}, \ldots, i_{k}$ be the non-critical rows of $F$. Assume that there are $c_{1}$ critical rows immediately above row $i_{1}$, and $c_{\ell+1}$ critical rows immediately below row $i_{\ell}$ for all $1 \leq \ell \leq k$. Denote $F^{\prime}$ the 01 -filling obtained from $F$ by removing all the critical rows and columns from $F$. Moreover, let $F^{\prime \prime}=\psi\left(F^{\prime}\right)$ and $x=x_{1} x_{2} \ldots x_{k+1}=\gamma^{-1}\left(F^{\prime \prime}\right)$. It is easily seen that $F^{\prime} \in \mathcal{N}_{3}(k), F^{\prime \prime} \in \mathcal{M}_{3}(k)$ and $x \in \mathcal{P} \mathcal{A}_{3}(k+1)$. Let $\delta^{\prime}(F)=$ $x_{1}^{c_{1}+1} x_{2}^{c_{2}} \ldots x_{k+1}^{c_{k+1}}$. It is apparent that we have $\delta^{\prime}(F) \in \mathcal{A}_{3}(n+1)$.

Property (b1) ensures that the inserted rows and columns in the construction of $\delta$ are exactly the removed rows and columns in the construction of $\delta^{\prime}$. Thus the map $\delta^{\prime}$ is the inverse of the map $\delta$. This implies that $\delta$ is bijection.

For example, let $x=001234345664$ be an ascent sequence in $\mathcal{A}_{3}(13)$. Then $x$ can be written as $0^{2} 1^{1} 2^{1} 3^{1} 4^{1} 3^{1} 4^{1} 5^{1} 6^{2} 4$. Let $x^{\prime}=0123434564$, which is an element of $\mathcal{P} \mathcal{A}_{3}(10)$. By applying the map $\gamma$ to $x^{\prime}$, we get a 01-filling

$$
F=\gamma\left(x^{\prime}\right)=\{(1,1),(2,2,),(3,3),(4,4),(5,4),(6,5),(7,6),(8,7),(9,6)\} \in \mathcal{M}_{3}(9)
$$

illustrated in Figure 5. Then by applying the map $\phi$ to $F$, we get a 01-filling $F^{\prime}$ as shown in Figure 5. Finally, we obtain a 01-filling $F^{\prime \prime} \in \mathcal{P}_{3}(12)$ by adding one zero row immediately above row 1 , one zero column immediately to the left of column 1 , two consecutive zero rows immediately below row 8 , and two consecutive zero columns immediately to the right of column 8, see Figure 5,

Combining Theorems 3.1 and 3.2, we get a new bijective proof of Conjecture 1.2 ,

Acknowledgments. This work was supported by the National Natural Science Foundation of China (11571320 and 11671366) and Zhejiang Provincial Natural Science Foundation of China ( LY15A010008). 


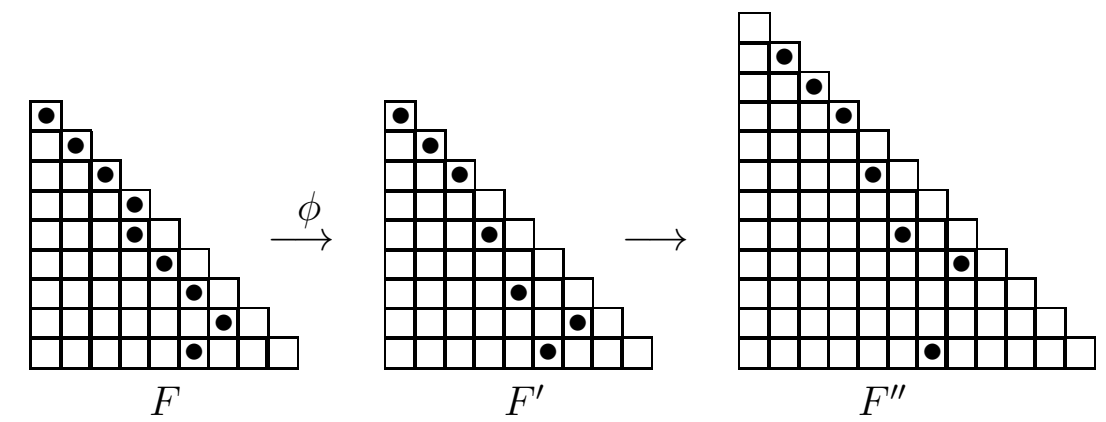

Figure 5: A 01-filling $F \in \mathcal{M}_{3}(9)$, a 01-filling $F^{\prime} \in \mathcal{N}_{3}(9)$ and a 01-filling $F^{\prime \prime} \in$ $\mathcal{P}_{3}(12)$.

\section{References}

[1] M. Bóna. Combinatorics of Permutations. CRC Press, 2004.

[2] M. Bousquet-Mélou, Four classes of pattern-avoiding permutations under one roof: generating trees with two labels, Electron. J. Combin. 9 (2003), $\sharp$ R19.

[3] M. Bousquet-Mélou, A. Claesson, M. Dukes, S. Kitaev, (2+2)-free posets, ascent sequences and pattern avoiding permutations, J. Combin. Theory Ser. A 117 (2010), 884-909.

[4] W.Y.C. Chen, A.Y.L. Dai, T. Dokos, T. Dwyer, B.E. Sagan, On 021-avoiding ascent sequences, Electron. J. Combin. 20(1) (2013), P76.

[5] W.Y.C. Chen, E.Y.P. Deng, R.R.X. Du, R.P. Stanley, C.H. Yan, Crossings and nestings of matchings and partitions, Trans. Amer. Math. Soc. 359 (2007), $1555-1575$.

[6] W.Y.C. Chen, J. Qin and C.M. Reidys, Crossings and nestings in tangled diagrams, Electron. J. Combin. 15 (2008), R86.

[7] S. Corteel, M. Martinez, C.D. Savage and M. Weselcouch, Patterns in inversion sequences I, Discrete Math. Theor. Comput. Sci. 18(2) (2016), $\sharp 2$.

[8] P. Duncan, E. Steingrímsson, Pattern avoidance in ascent sequences, Electron. J. Combin. 18 (2011), P226.

[9] D. Kim, Z. Lin, Refined restricted inversion sequence, arXiv:1706.07208v1. 
[10] S. Kitaev, Patterns in permutations and words, Springer Verlag (EATCS monographs in Theoretical Computer Science book series), 2011.

[11] C. Krattenthaler, Growth diagrams, and increasing and decreasing chains in fillings of Ferrers shapes, Adv. Appl. Math. 37 (2006), 404-431.

[12] M. van Leeuwen, Spin-preserving Knuth correspondences for ribbon tableaux, Electron. J. Combin. 12(1) (2005), R10.

[13] Z. Lin, Restricted inversion sequences and enhanced 3-noncrossing partitions, arXiv: $1706.07213 \mathrm{v} 1$.

[14] T. Mansour, M. Shattuck, Some enumerative results related to ascent sequences, Discrete Math. 315-316 (2014), 29-41.

[15] T. Mansour, M. Shattuck, Pattern avoidance in inversion sequences, Pure Math. Appl. 25 (2015), 157-176.

[16] M.A. Martinez, C.D. Savage, Patterns in Inversion Sequences II: Inversion Sequences Avoiding Triples of Relations, arXiv:1609.08106.

[17] M. Petkovsek, H.S. Wilf, D. Zeilberger, A=B, A K Peters Ltd., Wellesley, MA, 1996.

[18] A. Stoimenow, Enumeration of chord diagrams and an upper bound for Vassiliev invariants, J. Knot Theory Ramifications 7 (1998), 93-114.

[19] S.H.F. Yan, Ascent sequences and 3-nonnesting set partitions, European J. Combin. 39 (2014), 80-94. 